\title{
Radiation dose at CT coronary angiography: how low can we go?
}

\author{
${ }^{1} \mathrm{D}$ Eason, ${ }^{2} \mathrm{SJ}$ Leslie, ${ }^{3} \mathrm{AJ}$ Hince, ${ }^{1} \mathrm{JH}$ Miller
}

${ }^{1}$ Consultant Radiologist, Department of Radiology; ${ }^{2}$ Consultant Cardiologist, Department of Cardiology; ${ }^{3}$ Medical Physicist, Department of Medical Physics and Bioengineering, Raigmore Hospital, Inverness, Scotland, UK

ABSTRACT Computed tomography (CT) coronary angiography is now a widely available and reliable test accessible on basic $\mathrm{CT}$ platforms that can exclude coronary heart disease with confidence. It is fast, cheap and, if properly carried out by trained and accredited staff in carefully selected patients, useful information can be obtained with acceptably low radiation exposure in some cases.

KEYWORDS Radiation dose, coronary artery disease, CT scanning

DECLARATION OF INTERESTS No conflicts of interest declared.

\author{
Correspondence to JH Miller \\ Consultant Radiologist \\ Raigmore Hospital \\ Inverness IV2 3U] \\ Scotland
}

tel. +44 (0) I463704000

e-mail jmiller6@nhs.net

\section{CASE REPORT}

A 24 -year-old Caucasian nurse was referred to the rapid
access chest pain clinic with a history consistent with
ischaemic chest pain described as worsening upper
retrosternal discomfort on exercise (e.g. at the gym or
walking in the snow) which eased on stopping exercise.'
She was a slim non-smoker, her BMI was 22 , (subsequent
dimensions by computed tomography [CT] were anterior-
posterior [AP] diameter chest $17 \mathrm{~cm}$, lateral diameter
chest $26 \mathrm{~cm}$ ). The rest electrocardiogram (ECG) was
normal. There are no relevant risk factor scores for
conventional coronary disease in such young female
patients, where the likelihood of disease is very low. There
was sufficient concern expressed by the patient and
supervising clinician in this instance that imaging of the
coronary circulation was felt relevant to exclude such
disease or more likely congenital coronary anomaly.

After receiving a routine briefing on computed tomography coronary angiography (CTCA) technique and an oral beta blockade with $100 \mathrm{mg}$ oral metoprolol one hour before, she underwent a CTCA exam on a single source 64-slice CT scanner using prospective ECG gating with a stable sinus bradycardia of 58 beats per minute (bpm). Coronary calcium scoring was not obtained.A low-dose bolus tracking technique was used prior to scan acquisition, monitoring a $70 \mathrm{cc}$ injection of 350 milligrams of iodine molecules per millilitre $(\mathrm{mgl} / \mathrm{ml})$ strength ioversol with Optibolus software at 5 millilitres per second $(\mathrm{ml} / \mathrm{s})$ with a saline chaser of similar rate and volume. Excellent visualisation of the left sided cardiac structures was obtained with only three revolutions covering the heart. A 180 -degree filtered back projection reconstruction technique was used. Bismuth breast shields were not available.

Computed tomography scanning parameters were 80 peak kilovoltage $\left(k V_{p}\right), 450$ milliamperes $(m A), 0.35$ second rotation time, centering $70 \%$ of $R-R$ interval, zero padding and small cardiac filter. Enhancement in the aorta was $700+$ Hounsfield units $(\mathrm{HU})$. Dose length product (DLP) was 28 milligrays per centimetre (mGy.cm) and this equates to an effective dose of 0.8 millisievert ( $\mathrm{mSv}$ ) (specialist software ${ }^{2}$ was used to determine effective dose for the patient, based on tissue factors as per the recommendations of the International Commission on Radiological Protection [ICRP 103] $)^{3}$ with corrections for scanned region and patient size. ${ }^{4}$ In over four years and more than 900 scans this was our lowest DLP for CTCA. Post-processing on GE Advantage Windows v4.4 workstation using cardiac autoanalysis was uncomplicated.

No flow-limiting disease, anomalous anatomy or incidental finding was observed (Figures I and 2) and the patient was subsequently reassured. The patient was discharged from hospital and had no further presentation to hospital with chest pain over the subsequent six months.

\section{DISCUSSION}

Reliable CTCA has been available since 2004 when 64-slice CT scanners were first released. ${ }^{5}$ There has been understandable concern about increasing ionising radiation dose to the population due to $\mathrm{CT}$ exposure, particularly in sensitive organs such as the female breast. $^{6-10}$ Diagnostic image quality must always be balanced against radiation dose. There has been a desire to match and exceed radiation dose saving when compared to cardiac nuclear scintigraphy (I 2-20 mSv) and expert coronary catheter angiography (3-8 mSv). Newer CT scanners have improved temporal and spatial resolution due to second generation, dual tube capability and novel detector arrays respectively, although the line pair resolution of CT is still double that of catheter angiography at $0.4 \mathrm{~mm}$. Computed tomography $\mathrm{CA}$ has proven useful for the exclusion of coronary artery disease (CAD) in patients with low to medium clinical risk. ${ }^{5,11}$ It has a very high negative predictive value, although precise assessment of luminal stenosis is more 


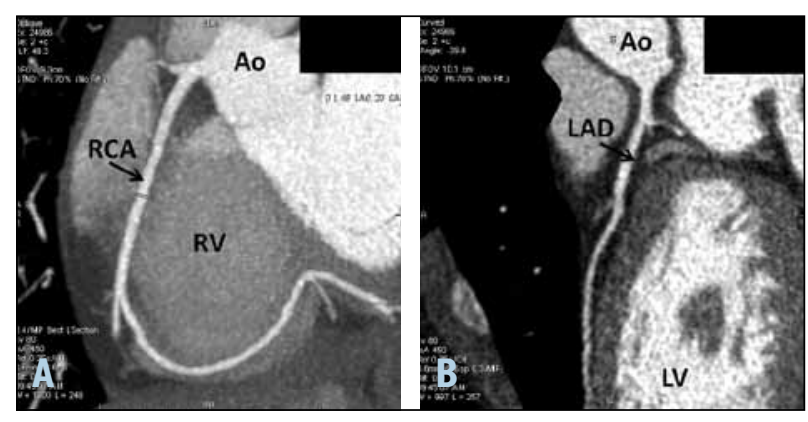

FIGURE IA Normal right coronary artery (RCA) reconstruction on low dose prospective electrocardiogram gated computed tomography coronary angiogram. Right ventricle (RV) and aortic root (Ao) are marked.

FIGURE IB Normal left anterior descending artery (LAD) reconstruction on low dose prospective electrocardiogram gated computed tomography (CT) coronary angiogram. Note CT parameters bottom left that indicate low dose technique. Left ventricle (LV) and aortic root (Ao) are marked.

challenging. It is also indicated to assess anomalous coronary anatomy, congenital heart disease, assist in surgical planning, aid anaesthetic fitness work-ups and follow-up coronary artery bypass graft (CABG) studies. The high negative predictive value of CTCA provides a useful triage tool for chest pain assessment although it has limitations in the morbidly obese and those with irregular tachyarrhythmias. Furthermore CT also excludes other pathology such as pulmonary embolism $(\mathrm{PE})$ and aortic dissection. Indeed, another diagnosis is elucidated on CT in 10-20\% of cases. Other nonanatomical tests are uncertain e.g. exercise tolerance testing (ETT) has a predictive value $\sim 50 \%$ for diagnosing coronary disease although it is nearer $\mathbf{8 0 \%}$ for diagnosing flow-limiting coronary disease. Myocardial perfusion scanning involves a relatively large radiation dose with a $20 \%$ false positive and negative rate and stress echo was not available in our centre at this time. We felt that CTCA offered an opportunity to exclude coronary artery disease with a high degree of certainty. At our hospital we refined our CTCA technique so that a tailored CTCA exam on an appropriate patient using a widely available platform produces a very low radiation burden, raising the prospect of wider acceptance of this reliable method of coronary assessment. Indeed $\sim 70 \%$ of our scans are between 2 and $4 \mathrm{mSv}$, although dose does depend on the size of the patient and achieving a sustained stable bradycardia.

To achieve such doses requires the presence of a dedicated physician at the time of the scan and an understanding of the multiple, complex CT factors and interactions to guide the $C T$ technologist. An effective dose of $0.8 \mathrm{mSv}$ is equivalent to 40 chest radiographs and lower than catheter angiography (3-8 mSv) and retrospective ECG gated CTCA at 6-25 mSv. In this case, we did not perform a CT coronary calcium score as the information was potentially irrelevant in this age group and the radiation dose would exceed that of the definitive angiogram. ${ }^{12}$ More advanced, newer scanners (>64-slice) would be capable of further significant dose reduction with the judicious application of optimal technique and newer iterative reconstruction algorithms. Bismuth latex breast shields promise the possibility of further breast dose reduction. Our CTCA scans cost $\mathrm{GB} \notin 250$ per patient including staff, hardware and consumable costs.

\section{CONCLUSIONS}

It is possible with meticulous individual tailoring of radiographic and intravenous $C T$ parameters to produce diagnostic CTCA images with very low radiation dose. The use of more modern CT scanners and software now available will only improve this situation and will likely lead to greater use of CTCA in the assessment of patients with chest pain.

\section{REFERENCES}

I National Institute for Health and Clinical Excellence (NICE). Chest pain of recent onset: assessment and diagnosis of recent onset chest pain or discomfort of suspected cardiac origin [Internet]. NICE; London: 2010 [cited 2012 May 16]. Available from: http://www. nice.org.uk/nicemedia/live//2947/47938/47938.pdf

2 Impactscan.org [Internet]. Imaging performance assessment of CT scanners. Impact 2009Ver. I.0. Available from: http://www.impactscan org/ctdosimetry.htm

3 The 2007 recommendations of the International Commission on Radiological Protection. ICRP Publication I03. Ann ICRP 2007; 37:I-332. http://dx.doi.org/10.1016/j.icrp.2007.II.001

4 Huda W,Tipnis S, SterzikA et al. Computing effective dose in cardiac CT. Phys Med Biol 20 I0; 55:3675-84. http://dx.doi.org/I0.1088/003 I9155/55/13/007

5 Budoff MJ, Dowe D, Jollis JG et al. Diagnostic performance of 64-multidetector row coronary computed tomographic angiography for evaluation of coronary artery stenosis in individuals without known coronary artery disease: results from the prospective multicenter ACCURACY trial. J Am Coll Cardiol 2008: 52:1724-32. http://dx.doi.org//0.1016/j.jacc.2008.07.03 I

6 Hausleiter J, Meyer T, Hermann $\mathrm{F}$ et al. Estimated radiation dose associated with cardiac CT angiography. JAMA 2009; 30I:500-7. http://dx.doi.org//0.100I/jama.2009.54

7 Gosling OE, Roobottom CA. Radiation exposure from cardiac computed tomography. JACC Cardiovasc Imaging 2010; 3:I20I-2. http://dx.doi.org/10.1016/j.jcmg.2010.09.006

8 Roobottom CA, Mitchell G, Morgan Hughes G. Radiation reduction strategies in cardiac computed tomographic angiography. Clin Radiol 2010; 65:859-67. http://dx.doi.org/10.1016/j.crad.2010.04.021

9 Achenbach S, Marwan M, Ropers D et al. Coronary computed tomography angiography with a consistent dose below ImSv using prospectively electrocardiogram-triggered high pitch spiral acquisition. Eur Heart J 20 I0; 31:340-6. http://dx.doi.org/I0.1093/eurheartj/ehp470

10 Earls JP, Berman EL, Urban BA et al. Prospectively gated transverse coronary $\mathrm{CT}$ angiography versus retrospectively gated helical technique: improved image quality and reduced radiation dose. Radiology 2008; 246:742-53. http://dx.doi.org// 0.I I48/radiol.2463070989

II British Society of Cardiovascular Imaging. Cardiac imaging report 2010 [Internet]. Glasgow: BSCl; 2010 [cited 2012 May 16] Available from: http://www.bsci.org.uk/downloads/doc_details/74cardiac-imaging-report-march-2010

12 Nance JW, Bamberg F, Schoepf UJ et al. Coronary atherosclerosis in African American and White patients with acute chest pain characterization with coronary CT angiography. Radiology 201I; 260:373-80. http://dx.doi.org/ I0.I I48/radiol. I I I I0I58 